EPJ Web of Conferences 37, 09035 (2012)

DOI: $10.1051 /$ epjconf/20123709035

(C) Owned by the authors, published by EDP Sciences, 2012

\title{
The Spectator-Induced Electromagnetic Effect on Meson Production in Nucleus-Nucleus Collisions at SPS Energies
}

\author{
Iwona Sputowska ${ }^{\mathrm{a}}$ and Andrzej Rybicki ${ }^{\mathrm{b}}$
}

H. Niewodniczanski Institute of Nuclear Physics, Polish Academy of Sciences, Cracow, Poland

\begin{abstract}
The electromagnetic interaction between the spectator system and the charged mesons produced in the course of the high energy heavy ion collision was studied experimentally and theoretically in earlier works [1,2]. This effect was found to result in very large distortions of the final state spectra of the produced mesons [3] and to bring new information on the space-time evolution of the non-perturbative meson production process [4]. In this paper a more extended analysis of this effect will be presented, including a comparative study between charged meson spectra produced in $\mathrm{Pb}+\mathrm{Pb}$ collisions as well as collisions of $\mathrm{Pb}$ ions with smaller nuclei. The experimental results will be compared with Monte Carlo simulations, giving a fair overall understanding of the interplay between the strong and the electromagnetic interaction in the heavy ion collision. A universal behaviour of charged meson spectra emerges from the above comparative study. This gives a unique chance of using the spectator charge as a tool to study the space-time evolution of the high energy nucleus-nucleus reaction.
\end{abstract}

\section{Introduction}

Processes occurring in nucleus-nucleus collisions include the participant zone and the highly charged nuclear remnant (spectator system). Interesting information about dynamics of the nuclear collision is brought by the analysis of electromagnetic interaction between produced particles and spectator protons. Theoretical and experimental studies on how this phenomenon impacts on charge meson distributions in $\mathrm{Pb}+\mathrm{Pb}$ collisions can be found in works [1,2]. These analyses have shown that the presence of spectator charge causes significant modification in the shape of meson spectra. This electromagnetic distortion is interrelated to the space-time scenario of collision and allows to draw conclusions about space-time evolution of particle production in nuclear reactions.

The aim of this paper is to extend the information about the spectator- induced electromagnetic effect. The main emphasis is put on comparison between charge $\pi$ meson spectra produced in two different types of nucleus-nucleus collisions $(\mathrm{Pb}+\mathrm{Pb}$ and " $\mathrm{Pb}+$ gas" reactions) and on results obtained from Monte Carlo simulations.

\section{Electromagnetic effect in peripheral $\mathrm{Pb}+\mathrm{Pb}$ and in $\mathrm{Pb}+$ gas collisions}

\subsection{Experiment and data}

The data come from the NA49 fixed target experiment at SPS at CERN. It was dedicated to study hadron-hadron, hadron-nucleus, nucleus-nucleus collisions (in particular $\mathrm{Pb}+\mathrm{Pb}$ reactions ) in the

a e-mail: iwona.sputowska@cern.ch

b All the experimental results and other experimental input used in this paper were obtained in the framework of the NA49 Collaboration. The theoretical calculations were performed in collaboration with A. Szczurek (H. Niewodniczanski Institute of Nuclear Physics, Polish Academy of Sciences).

This is an Open Access article distributed under the terms of the Creative Commons Attribution License 2.0, which permits unrestricted use, distribution, and reproduction in any medium, provided the original work is properly cited. 


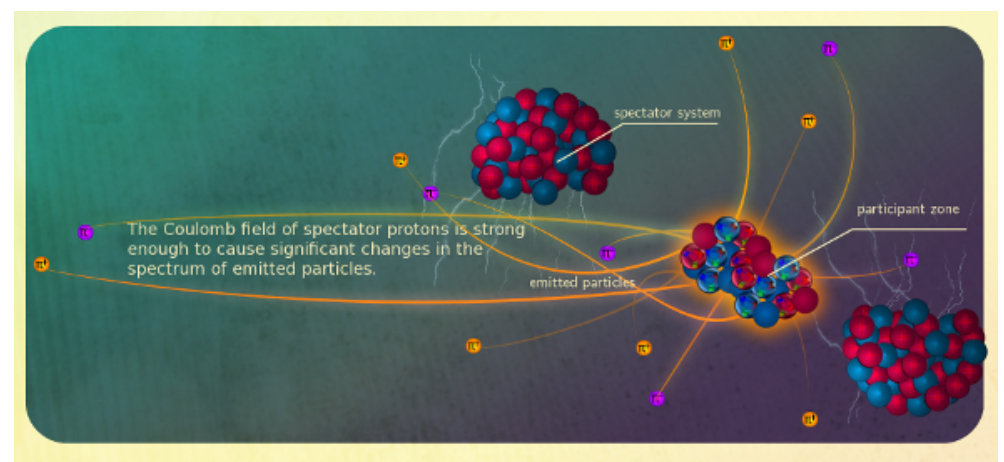

Fig. 1. Schematic picture of heavy ion collision at high energy.

range of beam energies from 20 to $158 \mathrm{GeV} /$ nucleon. We have compared charged $\pi$ meson spectra obtained from " $\mathrm{Pb}+$ gas" reactions and peripheral $\mathrm{Pb}+\mathrm{Pb}$ collisions at beam energy of $158 \mathrm{GeV} /$ nucleon. In this paper " $\mathrm{Pb}+$ gas" shall be understood as the collisions of large lead projectile with smaller nuclei of gas $(\mathrm{Pb}+\mathrm{N}, \mathrm{Pb}+\mathrm{O})$ in the vicinity of NA49 target (see [5] for more details discussion). The selection of peripheral $\mathrm{Pb}+\mathrm{Pb}$ sample was made by a cut $150-300$ on charged particle multiplicity. Only those of $\mathrm{Pb}+$ gas collisions were analysed in which the observed charged particle multiplicity was the same as the cut defining peripheral $\mathrm{Pb}+\mathrm{Pb}$ interactions.

\subsection{Charged pion spectra}
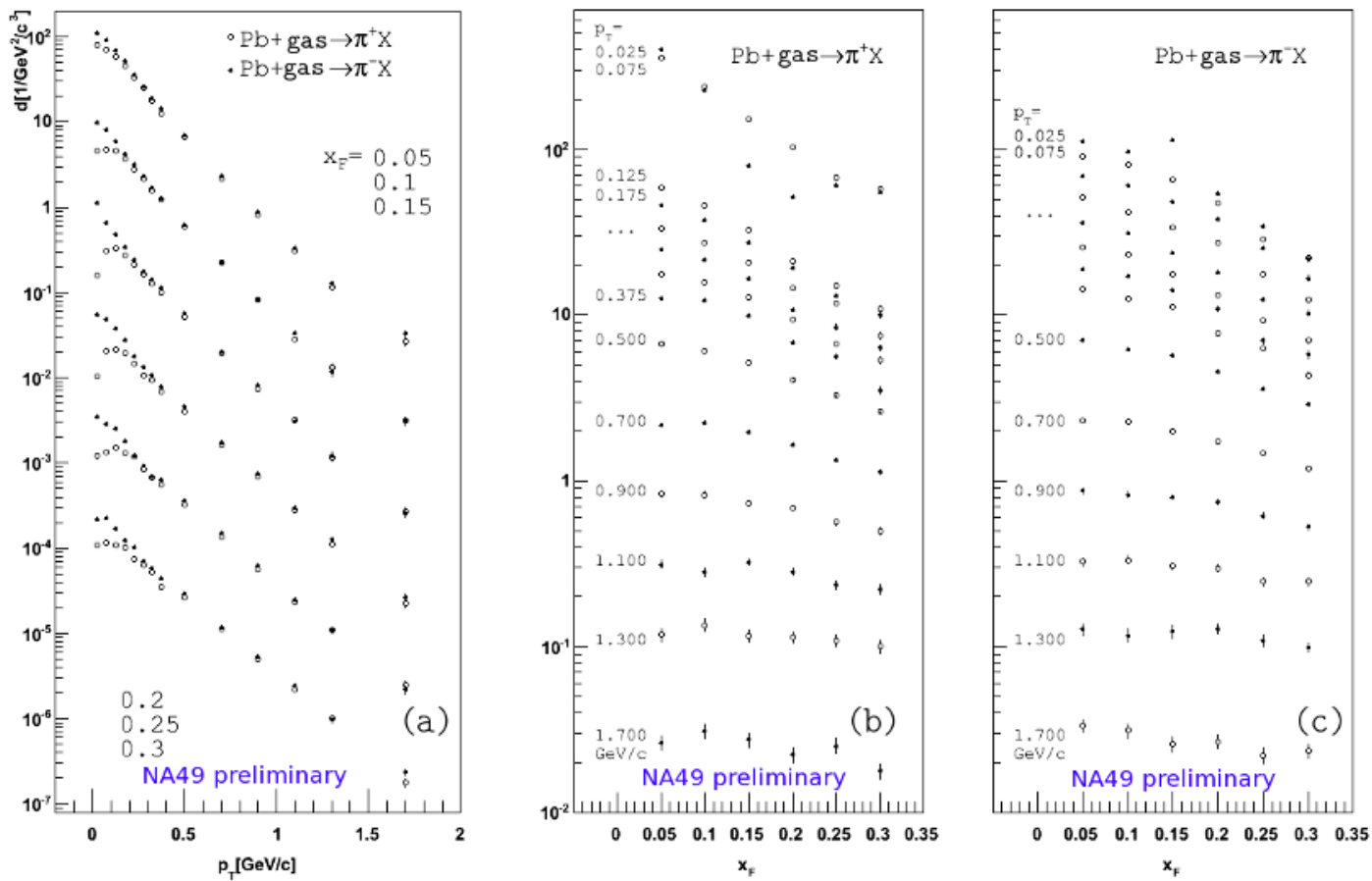

Fig. 2. Invariant density of charged pions produced in the projectile $(\mathrm{Pb})$ hemisphere of asymmetric $\mathrm{Pb}+\mathrm{gas}$ collisions drawn as a function of transverse momentum $p_{T}$ and Feynman variable $x_{F}=p_{L} / \sqrt{s_{N N}}$ (nucleonnucleon c.m.s). 
The measured results on charged $\pi$ meson production in $\mathrm{Pb}+$ gas collisions in projectile fragmentation region $\left(x_{F}>0\right)$ are shown in the Fig. 2 . The invariant density of $\pi^{+}$and $\pi^{-}$was plotted in a complementary way, as a function of $p_{T}$ and $x_{F}$. The evident dominance of negative over positive pions in the $p_{T}$ distribution chart (Fig. 2.(a)) can be explained as a result of the overlap of two factors: the isospin effect and the electromagnetic interaction between charged particles and spectator protons.

Firstly, particle production in nuclear collisions is affected by isospin. There is a surplus of neutrons over protons in $\mathrm{Pb}$ nucleus. The moderate excess of $\pi^{-}$over $\pi^{+}$observed for $p_{T}>0.3 \mathrm{GeV} / \mathrm{c}$ in the $p_{T}$ distribution chart can be explained by to the isospin content of the Pb projectile (see also [3]).

Significantly larger disparities in density of $\pi$ mesons are observed for low values of transverse momenta $\left(p_{T}<0.3 \mathrm{GeV} / \mathrm{c}\right)$. The biggest disproportion between $\pi^{-}$and $\pi^{+}$spectra occurs for $x_{F}=0.15=$ $\frac{m_{\pi}}{m_{N}}$ which is matching to pions moving longitudinally with the same velocity as spectator system. The observed decrease (increase) in density of positive (negative) pions in Fig. 2.(a) is a consequence of Coulomb repulsion (attraction) of $\pi^{+}\left(\pi^{-}\right)$by positively charged collision remnant of the projectile nucleus. This effect is also responsible for the very large difference evident in Fig. 2.(b),(c) between $x_{F}$ distributions of positive and negative $\pi$ mesons, measured at the lowest value of $p_{T}(0.025 \mathrm{GeV} / \mathrm{c})$.

\subsection{Comparative studies of $\pi^{+} / \pi^{-}$ratios}
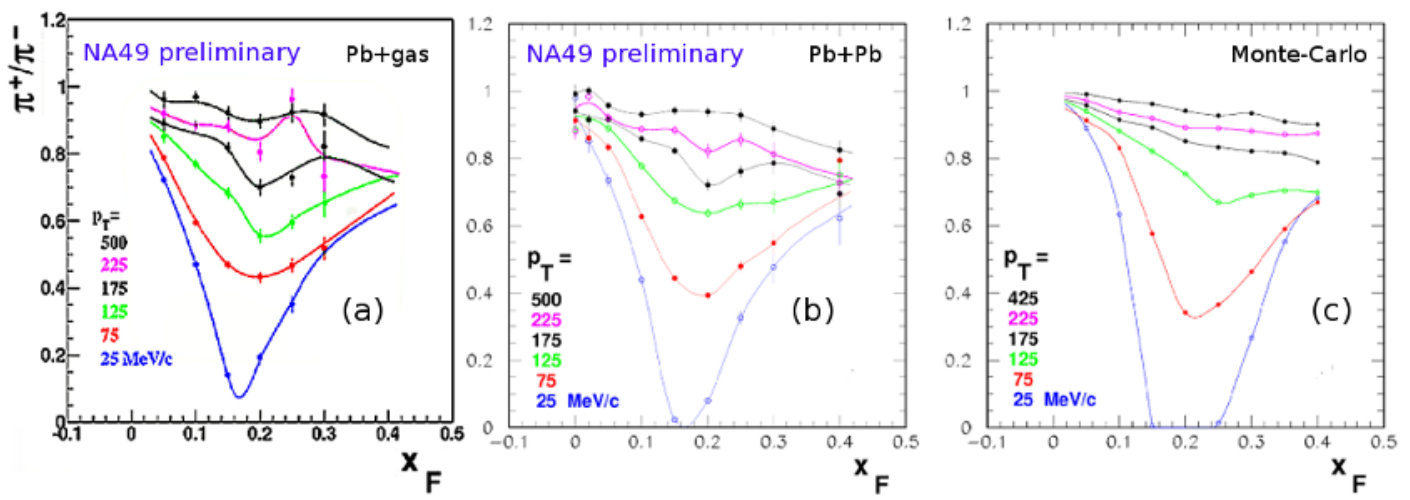

Fig. 3. $\pi^{+} / \pi^{-}$ratios drawn as a function of $x_{F}$ (nucleon-nucleon c.m.s.) at several values of $p_{T}$ for: (a) experimental data from $\mathrm{Pb}+$ gas collisions and (b) peripheral $\mathrm{Pb}+\mathrm{Pb}$ reactions [3] and (c) the results of $\mathrm{MC}$ simulation for peripheral $\mathrm{Pb}+\mathrm{Pb}$ collisions [3].

The comparative studies on electromagnetic distortion of $\pi$ meson spectra in $\mathrm{Pb}+\mathrm{Pb}$ and $\mathrm{Pb}+\mathrm{gas}$ collisions give the information on how the electromagnetic effect depends on reaction type and geometry.

Fig. 3.(a), (b) presents the ratio of charged pions $\left(\pi^{+} / \pi^{-}\right)$plotted as a function of $x_{F}$ for fixed values of $p_{T}$, respectively for $\mathrm{Pb}+$ gas and peripheral $\mathrm{Pb}+\mathrm{Pb}$ collisions. These experimental data are then compared with the results of Monte Carlo simulation for the latter reaction (Fig. 3.(c)). The corresponding theoretical model of the peripheral $\mathrm{Pb}+\mathrm{Pb}$ collisions, including the electromagnetic interaction, is described in detail in [1].

On each of the three panels, there is a characteristic structure as a function of $x_{F}$ and $p_{T}$ with sharp drop of $\pi^{+} / \pi^{-}$ratio for low values of $p_{T}$ around $x_{F}=0.15$ which disappears for higher values of transverse momenta. The quantitative agreement between the MC simulation and experimental data indicates that the observed "deformation" in charged pion ratios is indeed induced by the electromagnetic interaction of positively charged nuclear remnant with emitted particles. As described in work [1], the shape of electromagnetic deformation is sensitive to space-time evolution of the reaction. Therefore similarities in the shape between $\pi^{+} / \pi^{-}$ratios for peripheral $\mathrm{Pb}+\mathrm{Pb}$ and $\mathrm{Pb}+$ gas collisions indicate similarities in the space-time process of pion production. 


\section{Conclusions}

New data on pion production in collisions of the large $\mathrm{Pb}$ nucleus with smaller atomic nuclei have been obtained and presented in this work. The distributions of pions in latter collisions are evidently affected by the electromagnetic final state interaction between the electric charge of pions and that of spectator protons.

An overall similarity in this effect is apparent between the above " $\mathrm{Pb}+$ gas" reactions and peripheral $\mathrm{Pb}+\mathrm{Pb}$ collisions, indicating similarities in the space-time evolution of pion production in both collision types.

\section{Acknowledgement}

This work was supported by the Polish National Science Centre (funds granted on the basis of decision no. DEC-2011/03/B/ST2/02634).

\section{References}

1. A.Rybicki, A.Szczurek, Phys. Rev. C75 (2007) 054903

2. A.Rybicki, Acta Phys. Polon. B42 (2011) 867, and references therein

3. A.Rybicki, PoS(EPS-HEP 2009) 031

4. A.Szczurek, A.Rybicki, A.Z.Górski, J. Phys. G34 (2007) S827

5. A. Laszlo, Ph. D. Thesis, KFKI Research Institute for Particle and Nuclear Physics, Budapest, (2007) 